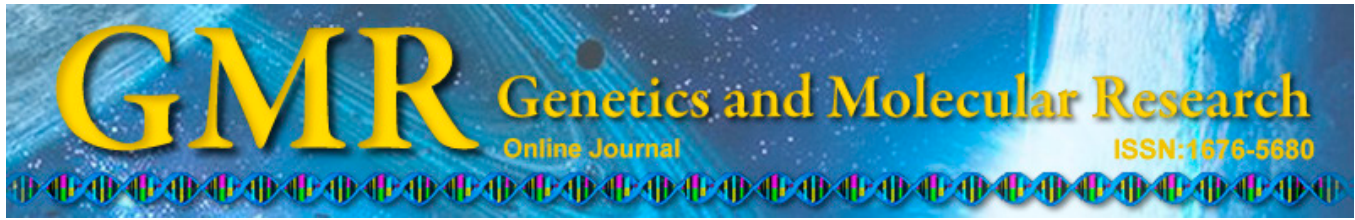

\title{
Distinct non-cerebrovascular risk factors for ischemic lacunar stroke and non-lacunar stroke: preliminary results
}

\author{
Y.F. Chen ${ }^{1}$, C.H. Luo ${ }^{1}$, Y.J. Liu ${ }^{2}$, W.H. Lu ${ }^{1}$ and B.R. Su${ }^{1}$ \\ ${ }^{1}$ Department of Nursing, Hunan University of Medicine, Huaihua, China \\ ${ }^{2}$ Department of Neurology, Zhujiang Hospital, Southern Medical University, \\ Guangzhou, China \\ Corresponding author: B.R. Su \\ E-mail: BinruSu@163.com
}

Genet. Mol. Res. 14 (2): 3170-3176 (2015)

Received March 10, 2014

Accepted November 23, 2014

Published April 10, 2015

DOI http://dx.doi.org/10.4238/2015.April.10.28

\begin{abstract}
Stroke is a non-communicable disease of increasing socioeconomic importance in aging populations. This study compared the risk factors implicated in two subtypes of ischemic stroke: lacunar stroke (LS) and non-lacunar stroke (NLS). A retrospective case control study was conducted on a total of 368 patients [220 cases $(59.8 \%)$ of NLS and 148 cases (40.2\%) of LS] with first-time onset of ischemic stroke. Multivariate logistic regression was performed to compare multiple non-cerebrovascular risk factors between the two groups. More patients with a history of diabetes were found in the NLS than the LS group (40.5 vs 26.4\%), and that both fasting glucose and HbA1C levels before the onset of stroke were higher in NLS than LS patients. Multivariate analysis revealed that patients with a history of diabetes were 1.57 times more likely to have NLS than LS $(\mathrm{OR}=1.57,95 \% \mathrm{CI}$ $=0.95-3.26)$. Moreover, male patients were more likely to develop
\end{abstract}


NLS than females $(\mathrm{OR}=1.46,95 \% \mathrm{CI}=0.79-2.69)$, and patients with elevated fibrinogen levels were 1.4 times more likely to develop NLS than $\mathrm{LS}(\mathrm{OR}=1.40,95 \% \mathrm{CI}=1.09-1.80)$. Additionally, patients who were heavy drinkers $(\mathrm{OR}=1.39,95 \% \mathrm{CI}=0.68-2.84)$ or smokers (OR $=1.62,95 \% \mathrm{CI}=0.91-2.89)$ were more likely to develop NLS than LS. Other risk factors, such as hypertension, dyslipidemia, age, and average blood pressure, did not differ between the two types of stroke. Thus, distinct non-cerebrovascular risk factors (male gender, long history of diabetes, elevated fibrinogen, heavy smoking, and heavy drinking) are associated with a higher risk of developing non-lacunar stroke than lacunar stroke.

Key words: Ischemic stroke; Lacunar stroke; Non-lacunar stroke; Male gender; Diabetes; Smoking

\section{INTRODUCTION}

Stroke is a non-communicable disease of increasing socioeconomic importance in aging populations. According to WHO, stroke is the second most common cause of mortality worldwide since 1990 and the third most common cause of mortality in more developed countries (http://www.who.int/). Large vessel disease accounts for about $50 \%$ of all types of ischemic stroke, small vessel disease for about $25 \%$, and cardioembolic disease for the remaining $25 \%$. Intracranial atherosclerosis is a primary biological basis of ischemic stroke (Amarenco et al., 2009).

Associations between risk factors and comorbid conditions with cerebrovascular disease have been analyzed mostly in epidemiologic and community-based studies for all categories of stroke, including initial ischemic stroke. About 60 to $80 \%$ of all ischemic strokes can be attributed to two groups of risk factors: 1) increasing blood pressure, blood cholesterol, cigarette smoking, carotid stenosis, and diabetes mellitus (atherosclerotic ischemic stroke), and 2) atrial fibrillation and valvular heart disease (cardiogenic ischemic stroke) (Amarenco et al., 2009). Other potential risk factors, although not proven, include raised apoB/apoA1 ratio, obesity, physical inactivity, psychosocial stress, and low fruit and vegetable intake (Kaul et al., 2000; Hankey, 2006).

Little research has been conducted to compare risk factors other than cerebral vascular stenosis between two subtypes of ischemic strokes: ischemic lacunar stroke (LS) and ischemic non-lacunar stroke (NLS). Vascular risk factors of LS and NLS relate directly to the underlying etiologies of the two subtypes (Kazui et al., 2000; Khan et al., 2012; Park et al., 2012). However, relatively little is known of the non-vascular risk factors contributing to ischemic stroke (Petty et al., 2000; Cokar et al., 2008; Prodan et al., 2008; Castilla-Guerra et al., 2009; Jackson et al., 2010; Zhang et al., 2011). Identifying the risk factors of ischemic stroke and the determinants of outcome (stroke recurrence and mortality) after onset of stroke is the basis for stroke prevention strategies. Therefore, the objective of this study was to determine and compare non-vascular risk factors contributing to LS and NLS in patients from a single center. 


\section{MATERIAL AND METHODS}

\section{Patients}

This is a retrospective study approved by the Medical Ethics Committee Institute Review Board (IRB) of our hospital. We included 368 patients with first time onset of ischemic stroke from September 2008 to October 2012. A total of 220 cases (59.8\%) of NLS and 148 cases $(40.2 \%)$ of LS were reviewed in this study.

\section{Data collection}

Patients were divided into two groups, LS and NLS, based on clinical presentation and neuro-radiological findings. LS was diagnosed in the presence of one of the five classic clinical syndromes associated with magnetic resonance imaging (MRI) evidence of a lacunar lesion measuring $\leq 1.5 \mathrm{~cm}$ in a territory supplied by deep or superficial small perforating arteries consistent with the clinical findings. Diagnostic criteria of NLS were clinical signs compatible with a hemispheric lesion associated with MRI evidence of a consistent cortical or subcortical lesion or with a lesion consistent with internal border zone infarct.

All diagnoses were based on Trial of ORG 10172 in Acute Stroke Treatment (TOAST) classification criteria (Adams Jr. et al., 1993).

We designed a case report form to collect the following data on admission to our Stroke Unit: age, gender, history of hypertension, diabetes mellitus, hyperlipidemia, smoking habits, alcohol abuse, and laboratory data.

\section{Statistical analysis}

We compared baseline characteristics between NLS patients and LS patients. An independent $t$-test was used for continuous variables, and the chi-square test or the Fisher exact test was used for categorical variables. Multivariate logistic regression was performed to compare non-vascular risk factors between the two groups by setting lacunar stroke as the reference group. $\mathrm{P}<0.05$ was considered to be statistically significant.

\section{RESULTS}

\section{Baseline characteristics of patients}

There were 368 patients in this study (Table 1), of which 220 patients had NLS and 148 had LS. The mean age of the patients was significant higher in the NLS group than in the LS group (65.6 vs 62.5 years, $\mathrm{P}=0.012$ ). Patients in the NLS group more frequently had a history of diabetes than did patients in the LS group ( $40.5 v s 26.4 \%, \mathrm{P}=0.005)$. NLS patients also had higher levels of fasting glucose and HbA1C before the onset of stroke. Serum fibrinogen was also significantly elevated in NLS compared to LS patients and more NLS than LS patients had a history of smoking and heavy drinking, although the difference was not significant. Interestingly, both systolic and diastolic blood pressures (SBP, DBP) were significantly lower in NLS than in LS patients. No differences were found in total cholesterol (TC), low-density lipoprotein (LDL), high-density lipoprotein (HDL) and triglyceride (TG) levels between the two types of stroke. 
Table 1. Baseline characteristics of patients.

\begin{tabular}{lrrr}
\hline & NLS $(\mathrm{N}=220)$ & LS $(\mathrm{N}=148)$ & $\mathrm{P}$ \\
\hline Age & $65.6(11.4)$ & $62.5(10.8)$ & 0.012 \\
Male [N (\%)] & $154(70.0)$ & $109(73.6)$ & 0.477 \\
Diabetes [N (\%)] & $89(40.5)$ & $39(26.4)$ & 0.005 \\
Smoking [N (\%)] & $101(45.9)$ & $58(39.3)$ & 0.201 \\
Drinking [N (\%)] & $45(20.5)$ & $22(14.9)$ & 0.173 \\
DBP & $85.8(11.2)$ & $88.1(11.4)$ & 0.074 \\
SBP & $155.1(22.1)$ & $164.1(23.5)$ & 0.052 \\
HDL & $1.1(0.6)$ & $1.1(0.5)$ & 0.630 \\
LDL & $3.1(1.0)$ & $2.9(0.9)$ & 0.230 \\
TG & $1.7(1.1)$ & $1.7(0.9)$ & 0.760 \\
TC & $5.1(1.2)$ & $4.9(1.1)$ & 0.200 \\
Fibrinogen & $3.9(1.1)$ & $3.6(0.8)$ & 0.029 \\
HbA1C & $7.2(2.1)$ & $6.6(1.8)$ & 0.024 \\
Glucose & $7.2(3.3)$ & $6.4(2.3)$ & 0.027 \\
HCA & $14.9(6.4)$ & $14.6(6.1)$ & 0.680 \\
\hline
\end{tabular}

HCA, homocysteic acid.

\section{Comparison of non-vascular risk factors between LS and NLS}

We ran a multivariate logistic regression analysis to more thoroughly assess the risk factors associated with NLS and LS. As shown in Table 2, multivariate analysis revealed that males were more likely to develop NLS than females $(\mathrm{OR}=1.46,95 \% \mathrm{CI}=0.79-2.69)$, and individuals with a history of diabetes were 1.57 times more likely to develop NLS than LS (OR = $1.57,95 \% \mathrm{CI}=0.95-3.26$ ). Elevated fibrinogen was associated more closely with the likelihood of having NLS than LS $(\mathrm{OR}=1.40,95 \% \mathrm{CI}=1.09-1.80)$. Additionally, patients who were heavy drinkers $(\mathrm{OR}=1.39)$ or smokers $(\mathrm{OR}=1.62)$ were more likely to have NLS than LS. Other risk factors examined in this study, such as hypertension, dyslipidemia, age, fasting glucose, and average blood pressure, did not differ between the two subtypes of ischemic stroke.

\begin{tabular}{|c|c|c|c|}
\hline & $\mathrm{OR}^{* *}$ & $95 \%$ CI lower & $95 \% \mathrm{CI}$ upper \\
\hline Age & 1.02 & 0.99 & 1.04 \\
\hline Male & 1.46 & 0.79 & 2.69 \\
\hline Diabetes & 1.57 & 0.95 & 3.26 \\
\hline Blood pressure & 0.98 & 0.96 & 3.26 \\
\hline $\mathrm{HDL}$ & 0.93 & 0.59 & 1.47 \\
\hline LDL & 1.04 & 0.73 & 1.47 \\
\hline TG & 1.00 & 0.76 & 1.25 \\
\hline $\mathrm{TC}$ & 1.11 & 0.80 & 1.54 \\
\hline Fibrinogen & 1.40 & 1.09 & 1.80 \\
\hline Glucose & 1.08 & 0.96 & 1.21 \\
\hline Drinking & 1.39 & 0.68 & 2.84 \\
\hline Smoking & 1.62 & 0.91 & 2.89 \\
\hline
\end{tabular}

*LS was the reference group; **adjusted OR.

\section{DISCUSSION}

The classification of acute ischemic stroke has a significant impact on patient care, secondary stroke prevention, and stroke research (Bejot et al., 2008; Amarenco et al., 2009). Nearly one quarter of ischemic strokes are caused by lacunar infarcts, resulting from the occlu- 
sion or leakiness of one of the small perforating arteries supplying the deep subcortical areas of the brain (Bejot et al., 2008). The responsible arterial pathology remains poorly understood, with proposed mechanisms including lipohyalinosis, arteriosclerosis, poor cerebral blood flow, vasospasm, and abnormal endothelial function (Kolominsky-Rabas et al., 2001; Khan et al., 2012). There is growing evidence to suggest that lacunar arteriopathy may differ from the atherothromboembolic processes that lead to occlusion of large intracranial and extracranial arteries, causing most other ischemic strokes (Jickling et al., 2012).

Lacunar stroke has been associated, at least in the short term, with a better outcome in terms of mortality and a lower risk for recurrent stroke than non-lacunar stroke (Arboix and Marti-Vilalta, 2009). In addition, recurrent cerebrovascular events following a lacunar ischemic stroke are more likely to be lacunar than non-lacunar, whereas recurrent cerebrovascular events following a non-lacunar stroke are more likely to be non-lacunar than lacunar (68 vs 15\%); importantly, these observations indicate that potentially different mechanisms are involved in the pathogenesis of these two stroke subtypes (Arboix and Marti-Vilalta, 2009).

Age, gender, hypertension, dyslipidemia, fasting glucose, and average blood pressure are considered risk factors in the development of ischemic stroke. Fang et al. (2013) reported that, in another Chinese patient population, hyperglycemia upon admission was an independent risk factor in non-lacunar stroke that was associated with poor outcome at 1 year. However, in lacunar stroke, hyperglycemia was not associated with functional outcome. Jackson et al. (2010) systematically reviewed studies comparing risk factors in patients with lacunar stroke versus non-lacunar stroke. They reported that 16 of 28 studies included risk factors in their ischemic stroke subtype definitions. Hypertension and diabetes appeared equally common in LS and NLS. Atrial fibrillation and carotid stenosis were associated more with NLS than LS (Jackson et al., 2010).

It was revealed that plasma fibrinogen level was significantly associated with coronary heart disease (CHD), stroke, and other causes of vascular and non-vascular mortality (Chuang et al., 2009; Chen et al., 2013). Unfortunately, very few studies report different risk of elevated fibrinogen between LS and NLS. Alvarez-Perez et al. (2011) conducted a prospective controlled clinical study involving 200 patients with ischemic stroke and 50 controls. Plasma levels of fibrinogen, D-dimer, C reactive protein and values for D-dimer/fibrinogen ratio and erythrocyte sedimentation rate were assessed over $48 \mathrm{~h}$ after admission. Patients with atherothrombotic stroke showed raised fibrinogen and erythrocyte sedimentation rate. Patients with lacunar showed moderated elevation of fibrinogen. Consistent with our results, Chuang et al. (2009) reported that fibrinogen independently predicted future ischemic stroke risk. Elevated fibrinogen tended to be more strongly associated with NLS than $\mathrm{LS}(\mathrm{HR}=1.40 ; 95 \% \mathrm{CI}=1.09$ to $1.80 ; \mathrm{P}=0.029)$.

The relationship between hyperglycemia and lacunar infarction remains controversial. Hyperglycemia in acute ischemic stroke is traditionally associated with a worsened outcome. Admission hyperglycemia has been demonstrated as a risk factor for non-lacunar infarction, especially in patients treated with thrombolysis (Paciaroni et al., 2009). Our results indicate that history of diabetes is a remarkable risk factor for NLS over LS despite the fact that there were no differences in blood glucose levels upon admission between NLS and LS groups.

Jia et al. (2012) reported that the prevalence of abnormal glucose regulation was high in Chinese patients with acute stroke, especially in patients with atherothrombotic infarction. Uyttenboogaart et al. (2007) examined the relationship between serum glucose measured within $6 \mathrm{~h}$ after stroke onset and functional outcome in 1375 ischemic stroke patients. The results of this study implied that hyperglycemia has a detrimental effect for non-lacunar stroke, 
whereas moderate hyperglycemia could be beneficial for lacunar stroke.

There are some limitations to the conclusions that can be drawn from these results. By their nature, observational studies such as this one may contain inevitable biasing and confounding factors that are not controlled for in the study design or data analysis. In this study, we conducted a chart review of electronic medical records. As a result, selection and recall bias may be major issues confounding the results of this study. However, there are advantages to obtaining data by chart review as compared to prospective data collection methods such as in a randomized clinical trial. These include the fact that it is a relatively inexpensive methodology for collecting data, the ability to capture conditions where there is a long latency between exposure and disease, the ability to study rare occurrences and the ability to generate hypotheses that can then be tested prospectively (vonKoss Krowchuk et al., 1995; Worster and Haines et al., 2004).

In conclusion, we have identified non-cerebrovascular risk factors that differentiate between the lacunar and non-lacunar subtypes of ischemic stroke. Specifically, male gender, a long history of diabetes, elevated fibrinogen levels, and a history of heavy smoking or heavy drinking are all associated with a higher risk of developing non-lacunar stroke than lacunar stroke.

\section{ACKNOWLEDGMENTS}

Research supported by the Science and Technology Program for Dongguan Higher Education, Science, Research and Health Care.

\section{REFERENCES}

Adams HP Jr, Bendixen BH, Kappelle LJ, Biller J, et al. (1993). Classification of subtype of acute ischemic stroke. Definitions for use in a multicenter clinical trial. TOAST. Trial of Org 10172 in acute stroke treatment. Stroke 24: 35-41.

Alvarez-Perez FJ, Castelo-Branco M and Alvarez-Sabin J (2011). Usefulness of measurement of fibrinogen, d-dimer, d-dimer/fibrinogen ratio, c reactive protein and erythrocyte sedimentation rate to assess the pathophysiology and mechanism of ischaemic stroke. J. Neurol. Neurosurg. Psychiatry 82: 986-992.

Amarenco P, Bogousslavsky J, Caplan LR, Donnan GA, et al. (2009). Classification of stroke subtypes. Cerebrovasc. Dis. 27: 493-501.

Arboix A and Marti-Vilalta JL (2009). Lacunar stroke. Expert Rev. Neurother. 9: 179-196.

Bejot Y, Catteau A, Caillier M, Rouaud O, et al. (2008). Trends in incidence, risk factors, and survival in symptomatic lacunar stroke in Dijon, France, from 1989 to 2006: A population-based study. Stroke 39: 1945-1951.

Castilla-Guerra L, Espino-Montoro A, Fernandez-Moreno MC, Lopez-Chozas JM (2009). Abnormal blood pressure circadian rhythm in acute ischaemic stroke: Are lacunar strokes really different? Int. J. Stroke 4: 257-261.

Chen YF, Su BR, Luo CH and Lu WH (2013). Risk factors for ischemic stroke due to large atherosclerosis and perforating artery disease. China J. Geriatr. Heart Brain Vessel Dis. 15: 1159-1161.

Chuang SY, Bai CH, Chen WH, Lien LM, et al. (2009). Fibrinogen independently predicts the development of ischemic stroke in a Taiwanese population CVDFACTS study. Stroke 40: 1578-1584.

Cokar O, Ozturk O, Aydemir T, Tiras R, et al. (2008). Transesophageal echocardiographic findings in patients with ischemic lacunar and nonlacunar stroke. J. Clin. Neurosci. 15: 246-252.

Fang Y, Zhang S, Wu B and Liu M (2013). Hyperglycaemia in acute lacunar stroke: A Chinese hospital-based study. Diab. Vasc. Dis. Res. 10: 216-221.

Hankey GJ (2006). Potential new risk factors for ischemic stroke: What is their potential? Stroke 37: 2181-2188.

Jackson CA, Hutchison A, Dennis MS, Wardlaw JM, et al. (2010). Differing risk factor profiles of ischemic stroke subtypes: Evidence for a distinct lacunar arteriopathy? Stroke 41: 624-629.

Jia Q, Zheng HG, Zhao XQ, Wang CX, et al. (2012). Abnormal glucose regulation in patients with acute stroke across China: prevalence and baseline patient characteristics. Stroke 43: 650-657. 
Jickling GC, Stamova B, Ander BP, Zhan X, et al. (2011). Profiles of lacunar and nonlacunar stroke. Ann. Neurol. 70: 477-485.

Kaul S, Venketswamy P, Meena AK, Sahay R, et al. (2000). Frequency, clinical features and risk factors of lacunar infarction (data from a stroke registry in South India). Neurol. India 48: 116-119.

Kazui S, Levi CR, Jones EF, Quang L, et al. (2000). Risk factors for lacunar stroke: A case-control transesophageal echocardiographic study. Neurology 54: 1385-1387.

Khan A, Kasner SE, Lynn MJ and Chimowitz MI (2012). Risk factors and outcome of patients with symptomatic intracranial stenosis presenting with lacunar stroke. Stroke 43: 1230-1233.

Kolominsky-Rabas PL, Weber M, Gefeller O, Neundoerfer B, et al. (2001). Epidemiology of ischemic stroke subtypes according to TOAST criteria: Incidence, recurrence, and long-term survival in ischemic stroke subtypes: A population-based study. Stroke 32: 2735-2740.

Paciaroni M, Agnelli G, Caso V, Corea F, et al. (2009). Acute hyperglycemia and early hemorrhagic transformation in ischemic stroke. Cerebrovasc. Dis. 28: 119-123.

Park JH, Ryoo S, Kim SJ, Kim GM, et al. (2012). Differential risk factors for lacunar stroke depending on the MRI (white and red) subtypes of microangiopathy. PLoS One 7: e44865.

Petty GW, Brown RD Jr, Whisnant JP, Sicks JD, et al. (2000). Ischemic stroke subtypes: A population-based study of functional outcome, survival, and recurrence. Stroke 31: 1062-1068.

Prodan CI, Joseph PM, Vincent AS and Dale GL (2008). Coated-platelets in ischemic stroke: Differences between lacunar and cortical stroke. J. Thromb. Haemost. 6: 609-614.

Uyttenboogaart M, Koch MW, Stewart RE, Vroomen PC, et al. (2007). Moderate hyperglycaemia is associated with favourable outcome in acute lacunar stroke. Brain 130: 1626-1630.

VonKoss Krowchuk H, Moore ML and Richardson L (1995). Using health care records as sources of data for research. $J$. Nurs. Meas. 3: 3-12.

Worster A and Haines T (2004). Advanced statistics: Understanding medical record review (MRR) studies. Acad. Emerg. Med. 11: 187-192.

Zhang B, Zhang W, Li X, Pu S, et al. (2011). Admission markers predict lacunar and non-lacunar stroke in young patients. Thromb. Res. 128: 14-17. 